\title{
A SHORT PROOF OF \\ TWO RECENTLY DISCOVERED INDEPENDENCE RESULTS USING RECURSION THEORETIC METHODS
}

\author{
E. A. CICHON
}

\begin{abstract}
Recently L. A. S. Kirby and J. Paris showed that a theorem of R. L. Goodstein cannot be proved in Peano's Arithmetic. We give an alternative short proof of their result, based only on well established results concerning recursion theoretic hierarchies of functions. A second, closely related result, duc to $F$. $S$. Beckman and $\mathrm{K}$. McAloon, is proved by the same means.
\end{abstract}

The motivation for this work was the recent result of Kirby and Paris in [2] that a certain number-theoretical statement, namely Goodstein's Theorem, is independent of Peano Arithmetic (Theorem 2 of this paper). We also include the proof of another similar result, due to Beckman and McAloon [1].

Process 1 . Given a natural number $N$, write it in base $x$ in the traditional way, i.e. as sums of powers of $x$. Increase the base of the representation by 1 , then subtract 1 from the new number thus obtained. Repeat the procedure of increasing the base by 1 and subtracting 1.

Process 2. This is the same as Process 1 except that at the outset $N$ is written in pure base $x$, i.e. $N$ is first written in base $x$, then so are the exponents and the exponents of exponents, etc.

EXAMPLE. $x=3, \quad N=20098=3^{9}+3^{5}+3^{4}+3^{4}+3^{2}+3^{0}=3^{3^{2}}+3^{3+2}+$ $3^{3+1}+3^{3+1}+3^{2}+3^{0}$. Changing base to 4 gives the new number $4^{4^{2}}+4^{4+2}+$ $4^{4+1}+4^{4+1}+4^{2}+4^{0}$. Subtracting 1 we get $4^{4^{2}}+4^{4+2}+4^{4+1}+4^{4+1}+4^{2}$.

These processes are said to terminate if the number 0 is eventually reached. Then we have

THEOREM 1. For any integer $N$ and base $x$, Process 1 eventually terminates, but this is not provable in Primitive Recursive Arithmetic.

TheOREM 2. For any integer $N$ and base $x$, Process 2 eventually terminates, but this is not provable in Peano Arithmetic.

The method of proof is the same for both theorems. We outline the proof of Theorem 2 which results from the following definitions and lemmas.

Received by the editors August 4, 1982. A handwritten version of the present paper appeared in the lecture notes of the AMS Summer Institute on Recursion Theory, Cornell Univ., Ithaca, N. Y., June 28-July 16, 1982.

1980 Mathematics Subject Classification. Primary 03F30; Secondary 03F15, 03D20.

Key words and phrases. Goodstein's theorem, pure number base, cantor normal form, fundamental sequences, slow-growing hierarchy, Hardy hierarchy. 
DEFINITION 1. We assume familiarity with the cantor normal form for ordinals below $\varepsilon_{0}, \operatorname{CNF}\left(\varepsilon_{0}\right)$, where $\varepsilon_{0}$ is the first ordinal $\alpha$ such that $\alpha=\omega^{\alpha}$.

Definition 2. Fundamental sequences to limit ordinals in $\operatorname{CNF}\left(\varepsilon_{0}\right)$. These are given by: $\{\omega\}(x)=x:\left\{\Gamma+\omega^{\alpha+1}\right\}(x)=\Gamma+\omega^{\alpha} \cdot x$; and if $\alpha$ is a limit ordinal, $\left\{\Gamma+\omega^{\alpha}\right\}(x)=\Gamma+\omega^{\{\alpha\}(x)}$. For details see Wainer [3].

This particular choice of fundamental sequences is necessary for the proofs of Theorems 1 and 2. With a different choice Lemma 1 below would fail.

Definition 3. The slow-growing hierarchy. For $x<\omega, \alpha \in \operatorname{CNF}\left(\varepsilon_{0}\right), G_{x}(0)=0$; $G_{x}(\alpha+1)=G_{x}(\alpha)+1$; and if $\alpha$ is a limit ordinal, $G_{x}(\alpha)=G_{x}(\{\alpha\}(x))$.

Definition 4. For $x<\omega, \alpha \in \operatorname{CNF}\left(\varepsilon_{0}\right), P_{x}(0)=0 ; P_{x}(\alpha+1)=\alpha$; and if $\alpha$ is a limit ordinal, $P_{x}(\alpha)=P_{x}(\{\alpha\}(x))$.

$P_{x}$ is a function which "subtracts" 1 from an ordinal $\alpha$.

Note that when $\alpha$ has cantor normal form $\omega^{\alpha_{1}}+\cdots+\omega^{\alpha_{k}}$ and $\beta$ has cantor normal form $\omega^{\beta_{1}}+\cdots+\omega^{\beta_{j}}$ and $\alpha_{\kappa} \geqslant \beta_{1}$ then the cantor normal form of $\alpha+\beta$ is just the concatenation of the cantor normal form of $\alpha$ with that of $\beta$. When $\alpha$ and $\beta$ are such that this holds, we have

LEMMA 1. (1) $G_{x}(\alpha+\beta)=G_{x}(\alpha)+G_{x}(\beta)$.

(2) $G_{x}\left(\omega^{\alpha}\right)=x^{G_{1}(\alpha)}$.

Proof. Induction on $\beta$ for (1), on $\alpha$ for (2).

In what follows we omit parentheses when composing functions.

Lemma 2. For $x<\omega, \alpha \in \operatorname{CNF}\left(\varepsilon_{0}\right), G_{x} P_{x}(\alpha)=P_{x} G_{x}(\alpha)\left(=G_{x}(\alpha)-1\right)$.

Proof. Induction on $\alpha$.

REMARK 1. Because of Lemma 1 we see that $G_{x}(\alpha)$ is obtained by taking the representation of $\alpha$ in $\operatorname{CNF}\left(\varepsilon_{0}\right)$ and replacing $\omega$ throughout by $x$.

Remark 2. Following Remark 1 , if $N$ is written in pure base $x$ and $x$ is then replaced throughout by $\omega$ we obtain an ordinal $\alpha$ in $\operatorname{CNF}\left(\varepsilon_{0}\right)$ and we have $N=G_{x}(\alpha)$.

We can now express Process 2 as follows: Write $N$ in pure base $x$ to obtain an ordinal $\alpha$ (as described in Remark 2), so $N=G_{x}(\alpha)$. Change base to $x+1$ obtaining $G_{x+1}(\alpha)$. Subtract 1, i.e. form $P_{x+1} G_{x+1}(\alpha)$ which by Lemma 2 is $G_{x+1} P_{x+1}(\alpha)$. Change base to $x+2$ obtaining $G_{x+2} P_{x+1}(\alpha)$. Subtract 1, forming $P_{x+2} G_{x+2} P_{x+1}(\alpha)$, which by Lemma 2 is $G_{x+2} P_{x+2} P_{x+1}(\alpha)$, etc.

It can easily be established by induction on $\alpha \in \operatorname{CNF}\left(\varepsilon_{0}\right)$ that when $x \neq 0$ then $G_{x}(\alpha)=0$ iff $\alpha=0$, and so it should now be clear that Theorem 2 is equivalent to: "For all $\alpha$ in $\operatorname{CNF}\left(\varepsilon_{0}\right)$ and for all $x \neq 0$, there exists $y>x$ such that $P_{y} P_{y-1}$ $\cdots P_{x+2} P_{x+1}(\alpha)=0$ " is true but not provable in Peano Arithmetic.

To prove this we introduce one more function hierarchy, the Hardy functions: For $x<\omega, \alpha \in \operatorname{CNF}\left(\varepsilon_{0}\right), H_{0}(x)=x ; H_{\alpha+1}(x)=H_{\alpha}(x+1)$; and if $\alpha$ is a limit ordinal, $H_{\alpha}(x)=H_{\{\alpha\}(x)}(x)$.

By the work of Wainer in $[3,4], H_{\varepsilon_{0}}$ is not provably recursive in Peano Arithmetic and $H_{\omega}$ is precisely a version of the Ackermann function and so is not provably recursive in Primitive Recursive Arithmetic. 
Theorem 2 now follows from: If $\alpha \in \operatorname{CNF}\left(\varepsilon_{0}\right)$ with $\alpha \neq 0$ and $x \neq 0$, by induction on $\alpha$ we have $\mu y\left[P_{y} P_{y-1} \cdots P_{x+2} P_{x+1}(\alpha)=0\right]=H_{\alpha}(x+1)-1$.

The proof of Theorem 1 is identical. We only need to observe that when $N$ is written in traditional base $x$ and then $x$ is replaced throughout by $\omega$, the ordinal thus obtained is smaller than $\omega^{\omega}$.

\section{REFERENCES}

1. F. S. Beckman and K. Mc Aloon. A direct proof of a result of Goodstein-Kirhi-Paris. Lecture Notes, AMS Summer Institute on Recursion Theory. Cornell Univ., Ithaca. N. Y.. June 28-July 16. 1982.

2. L. A. S. Kirby and J. Paris, Accessible independence results for Peano Arithmetic. Bull. London Math. Soc. 14 (1982), 285-293

3. S. S. Wainer. A classification of the ordinal recursite functions, Arch. Math. Logik Grundlagenforsch. 13 (1970). 136-153.

4. . Ordinal recursion and a refinement of the extended Grzegorcilk hierarchl. J. Symbolic Logic 37 (1972). 281-292.

Department of Mathematics. Penn State University. University Park. Pennsylvania 16802 